\title{
Ameliorative effects of Lipitor against hypercholesterolemia-induced nephrotoxicity in rats and their breast-feeding young
}

\author{
Abdelalim A. Gadallah ${ }^{1,2}$ \\ ${ }^{1}$ Zoology Department, Faculty of Science, Mansoura University, Egypt \\ ${ }^{2}$ Preparatory Year Deanship, Jazan University, Kingdom of Saudi Arabia
}

\section{Article history}

Received: 14 Feb, 2014

Revised: 20 Mar, 2014

Accepted: 24 Mar, 2014

\begin{abstract}
The aim of this study was to find the ameliorative effect of Lipitor in nephrotoxicity resulting from hypercholesterolemia in mothers and their young. Eighty fertile male and virgin Wistar female rats were used in the present study. After mating and confirmation of pregnancy, the rats were arranged into four groups (10 per group) as follows: control; $\mathrm{G} 1$, Lipitor-treated group ( $2 \mathrm{mg} / \mathrm{kg}$ body weight, orally administered every other day from the $6^{\text {th }}$ day of gestation until 14 and 21 days postnatal); G2, experimental hypercholesterolemic and G3, Hypercholesterolemic and Lipitor-treatment. At the end of treatments, kidney and femoral epiphysis of both control and experimental mother rats and their offspring at 7 and 14 days were processed for histological study. The results revealed that mothers exhibited tubulo-necrosis, glomerulonephritis and periglomerular and tubular leukocytic infiltration. Maternal nephrotoxicity was reflected in the renal picture of breast-feeding young at 2 and 3 weeks. Also, examining femoral epiphysis of breast-feeding rat maternal fed on a hypercholesterolemic diet exhibited a comparative reduction of epiphysis and derangement of cartilage column and widened of cartilage stromata. The increased damage and degeneration of cartilage cells and comparative reduction of epiphyseal cartilage may lead to softening of bone, decreased its growth rate and increased incidence of fractures. Furthermore Lipitor-treatment alone or in combination with hypercholesterolemia improved femoral epiphysis and renal tissue to some extent and not equal to normal ones. In conclusion, hypercholesterolemia altered renal morphology which might have affected synthesis of essential elements of bone formation leading to defective bone growth in their offspring. Lipitor-treatment improved maternal renal structure which also resulted in improved bone growth of their young.
\end{abstract}

Keywords: Amelioration, lepitor, nephrotoxicity, hypercholesterolemia, mothers, youngs

To cite this article: Gadallah AA, 2016. Ameliorative effects of Lipitor against hypercholesterolemia-induced nephrotoxicity in rats and their breast-feeding young. Res. Opin. Anim. Vet. Sci., 6(2): 53-58.

\section{Introduction}

It is well known that lifestyle and diet play a role in the development of kidney disease. Several studies indicated that abnormalities in lipid metabolism can often accompany and exacerbate renal diseases (SanzRosa et al., 2001; Ghosh et al., 2015). Hypercholesterolemia is well-known to be an independent risk factor for

*Corresponding author: Abdelalim A. Gadallah, Preparatory Year Deanship, Jazan University, Kingdom of Saudi Arabia; E-mail: abdelalimg@gmail.com; aagadallah@jazanu.edu.sa 
renal injury (Oda and Keane, 1999) and to aggravate the pathogenesis of a variety of clinical and experimental renal diseases (Stulak et al., 2001; Kivipelto et al., 2001; Sucajtys-Szulc et al., 2016). High cholesterol diet (HCD) was found to increase blood pressure and induced renal injury (Zou et al., 2003). Moreover, many accumulating evidences support the idea that HCD exacerbates kidney damage in animal models of kidney diseases (Mori and Hirano, 2012). More recent studies have demonstrated the role of oxidized high-density lipoprotein (HDL) and lowdensity lipoprotein (LDL) particles in the progression of kidney diseases (Gyebi et al., 2012).

In patients with reduced kidney function, this pattern is characterized by increased triglycerides, apolipoprotein CIII and remnant particles as well as decreased HDL cholesterol and apolipoprotein A1. Low-density lipoprotein cholesterol and apolipoprotein $\mathrm{B}$ are generally reported to be similar to those of healthy individuals (Keane et al., 2011; Arnadottir, 2012). Endothelium-dependent vasodilation was found to impair hypercholestero-lemia (Andrade et al., 1998; Wolfort et al., 2008). Abnormalities in lipid metabolism induced by HCD were reported to exacerbate renal diseases via oxidative stress pathways (Al-Rejaie et al., 2012).

Lipid-lowering agents are basic drugs for primary and secondary prevention of cardiovascular diseases and have now been in use for more than 4 decades. Over the past 5 years, large trials of several statins and other lipid-lowering interventions provided important information on the efficacy of these drugs in various risk groups and settings. Large-scale meta-analyses of randomized controlled trials are important to document the overall benefit of interventions and to explore the effect of clinically relevant outcomes in important subgroups (Studer et al., 2005).

The present study aimed to illustrate the effects of cholesterol overload on maternal nephrotoxicity and long bone damage and renal damage in new born rats and the ameliorating role of Lipitor.

\section{Materials and Methods}

\section{Experimental design}

Eighty fertile male and virgin female rats of Wistar strain (Rattus norvegicus) (at a ratio of 1 male and 3 females) weighing approximately $125 \mathrm{~g}$ were obtained from the Hellwan Breeding Farm, Ministry of Health, Egypt. They were housed in cages with good ventilation on a $12 \mathrm{~h}$ light and dark cycle. Females were mated overnight and zero date of gestation was determined the next morning by the presence of sperm in the vaginal smear. The pregnant rats were arranged into four groups $(\mathrm{n}=10$ per each) as follows: $\mathrm{G} 0$, control; G1, Lepitor-treated (Pfizer pharmaceutical,
Egypt) group ( $2 \mathrm{mg} / \mathrm{kg}$ body weight orally administered in $0.2 \mathrm{ml}$ saline every other day from the $6^{\text {th }}$ day of gestation until 14 and 21 days postnatal); G2, experimental hypercholesterolemic and G3, hypercholesterolemic and Lipitor-treated. Pregnant rats of both control and experimental groups were killed by light diethyl ether anesthesia and their young were killed at 2 and 3 weeks of age.

Hypercholesterolemic group was fed a hypercholesterolemic diet according to Enkhmaa et al. (2005). The hypercholesterolemic diet was composed of 3\% cholesterol, $1 \%$ thiouracil, $2 \%$ cholic acid, and $15 \%$ cocoa butter in accordance with the standard diet formula. The rats were fed for 6 weeks before the onset of gestation as well as throughout gestation and lactation period until 14 and 21 days postnatal. The control group was supplied a standard diet free from cholesterol and fat diet components.

At 7 and 14 day post-partum, offspring were anesthetized with diethylether and their kidneys and femoral epiphysis were incised immediately, fixed in $10 \%$ phosphate-buffered formalin $(\mathrm{pH} 7.4)$, and processed for histological investigation. At 14 days post-partum, mother rats of all groups were sacrificed by diethyl ether and their kidneys were removed, fixed in $10 \%$ formalin. Both tissues were dehydrated in ascending grades of ethyl alcohol, cleared in xylene and mounted in molten paraplast $58-62^{\circ} \mathrm{C}$. Serial $5 \mu \mathrm{m}$ histologic sections were cut and stained with Hematoxylin and Eosin and examined under bright field Olympus light microscope (Optical Industrial Company, Japan).

\section{Results}

\section{Kidney}

Mother rats: The histological study of the normal renal cortex is composed of numerous glomerulus surrounded by Bowman's capsule. The proximal and distal convoluted tubules showed normal lining epithelium and tubular lumina (Fig. $1 \mathrm{~A}$ ).

The kidneys Lipitor-treated mothers rats showed degenerated glomeruli and interstitial inflammatory cell infiltration. The proximal convoluted tubule exhibited edematous changes with apparent necrosis of their epithelial lining cells and deposition of hyaline substances within tubular lumina (Fig. $1 \mathrm{~B}$ ).

Hypercholesterolemic mother rats showed generalized peri-glomerular lymphocytic infiltration. There was lymphocytic infiltration and fibrosis within the renal interstitium. There was also marked the appearance of degenerated renal corpuscles, and increase deposition of red blood corpuscle within some of the glomerular tufts (Fig. 1C-C1).

The hypercholesterolemic group that was treated with Lipitor showed features of healing i.e., normal 
glomerulus, absence of inflammatory cells, normal basement membrane and capillaries and a decrease in hyaline deposit. The tissue necrosis was also observed to be reduced (Fig.1 D-D1).

Breast-feeding offspring: In control, 14 d-old rats, the renal cortex showed a normal uniform appearance of the cortex with the disappearance of all immature forms. The renal corpuscles appeared spherical in shape with evident Bowman's spaces. The renal corpuscles in the superficial cortex were smaller with compact capillary tufts, while those of the deeper cortex appeared larger with increased size with prominent capillary tufts (Fig. 2 A). In contrast, pups maternallytreated with Lipitor showed slight pathological alterations confined mainly in degeneration of the glomerular tuft (Fig. 2B).

Compared with the control group, numerous abnormalities were observed in the kidneys of pups of hypercholesterolemic-treated groups. Massive glomerular and tubular necrosis was detected (Fig. 2C). In hypercholesterolemic group-treated with Lipitor, a densed peri-tubular inflammatory cellular infiltration with the formation of granulomatous lesions, degeneration of cells in the epithelial lining associated with marked missing of the lumen and swollen cells occupying the entire Bowman's capsule space were observed (Fig. 2D).

At day 21 in control, the renal cortex revealed the presence of numerous developed renal corpuscles having prominent glomeruli and Bowman's capsules. The parietal layer of the Bowman's capsule was formed of the flat cells. Proximal and distal convoluted tubules were also seen intact (Fig. 3A1).

Lipitor-treatment revealed marked alterations in both renal corpuscles and renal tubules. Degeneration of glomerular tuft and reddish-coloration of tubular lumina were also detected (Fig. 3B1).

In contrast, the hypercholestyerolemic-treated group showed massive degeneration of epithelial cells lining the tubules, with marked distortion and the absence of their tubular lumina. The inflammatory cells densely accumulated between the renal tubules and dispersed in peri-glomerular regions (Fig. $3 \mathrm{C} 1$ ).

On the other hand, hypercholesterolemic-groups treated with Lipitor showed marked amelioration of the majority of renal tubules and glomeruli, but still some regions illustrated signs of pathological alterations (Fig. 3 D1).

\section{Femoral epiphysis}

In control at day 21, offspring possessed normal epiphysis composed of regularly arranged cartilage column infiltrated by thin cartilage stroma. The femoral epiphyseal line appeared irregular (Fig. 4 A). Lipitortreatment disrupted cartilage column arrangement associated with degeneration of cartilage cells as well as regeneration of other ones at the peripheral margin near the epiphyseal line. The drug exerted toxicity causing a marked reduction of the epiphyseal cartilage (Fig. 4B). On the other hand, hypercholesterolemic revealed marked reduction of epiphyseal cartilage associated with degeneration of cartilage cells and widened stroma. Fine collection of chondrocytes was distributed in haphazard fashion within the hyaline matrix of the epiphyseal cartilage (Fig. 4 C\&D). The experimental hypercholesterolemic group treated with Lipitor possessed newly formed cartilage cells with small lacunae, but cartilage columns still lacked ordinary arrangement (Fig. 4E).

\section{Discussion}

Hypercholesterolemia is the main cause of atherosclerosis, which affect large and medium sized arteriole walls interfere with cardiovascular disease and represent one of the causes of death (Badimon and Vilahur, 2014).

The present findings revealed that feeding mother rats for 6 weeks prior to conception and throughout gestation and breast-feeding period exhibited nephrotoxicity of tubulo-necrosis, glomerulonephritis and periglomerular and tubular leukocyte infiltration in mothers. Maternal nephrotoxicity was reflected in the renal picture of breast-feeding young ones at 2 and 3 weeks.

Similar findings of hypercholesterolemia associated nephrotoxicity were reported elsewhere (Maschio et al., 1991; Greco and Breyer 1997). Also, Long-term hypercholesterolemia appeared to be associated with contrast media-induced acute renal failure (Yang et al., 2004 \& 2012). Feeding experimental guinea pigs (French et al., 1967) and rats (Kim et al., 2009) in the hypercholesterolemic diet were found to increase focal and segmental glomerulonephrosis.

Hypercholesterolemic associated nephrotoxicity may be attributed to increased cholesterol accumulation in macrophages and other immune cells initiating inflammatory responses, such as augmentation of Toll-like receptor (TLR) signalling, inflammasome activation, and the production of monocytes and neutrophils in the bone marrow and spleen (Tall and Yvan-Charvet, 2015). Also, it was found that hyperlipidemia increased the rates of monocyte reactive oxygen species (ROS) generation associated with increased inflammatory mediators, such as TNF $\alpha$ and IL- $1 \beta$ facilitated renal damage (Oberle et al., 1992).

Examining femoral epiphysis of breast-feeding rat maternally fed on a hypercholesterolemic diet exhibited comparative reduction of epiphysis and derangement of 
M

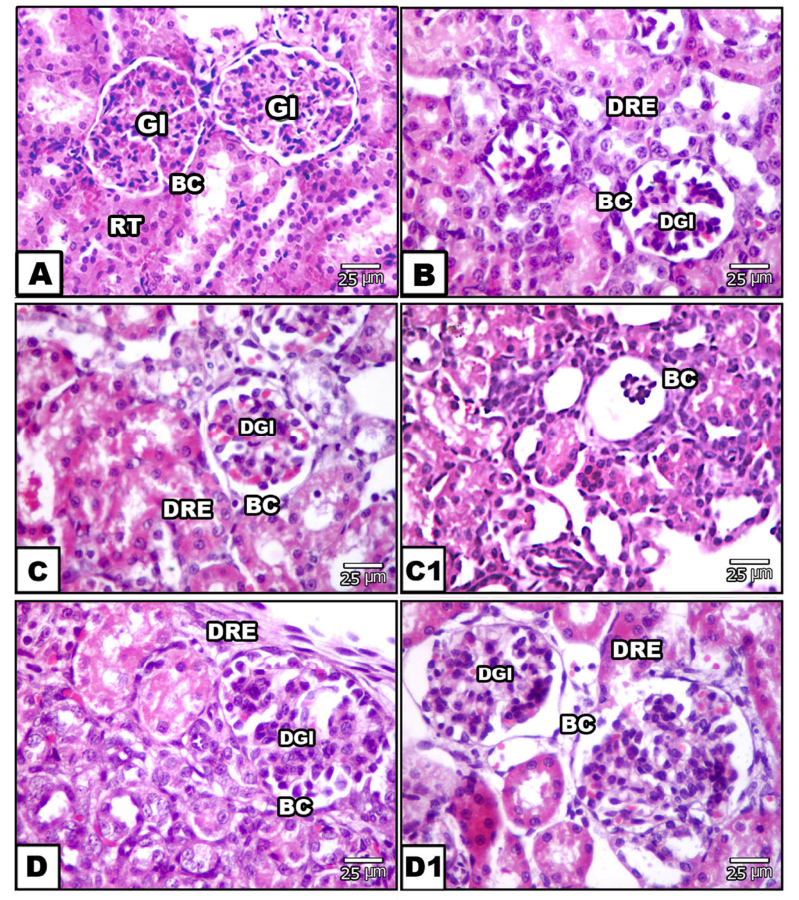

Fig. 1: Photomicrographs of histological sections of renal tissues of mother rats. A. Control, B: Lepitortreatment. C-C1: Hypercholesterolemia-treatment. D-D1: Hypercholesterolemia and lepitor-treatment. H\&E. Abbreviations; BC: Bowman's capsule; DGI: degenerated glomeruli, DRE: Degenerated renal epithelium; GI: Glomeruli

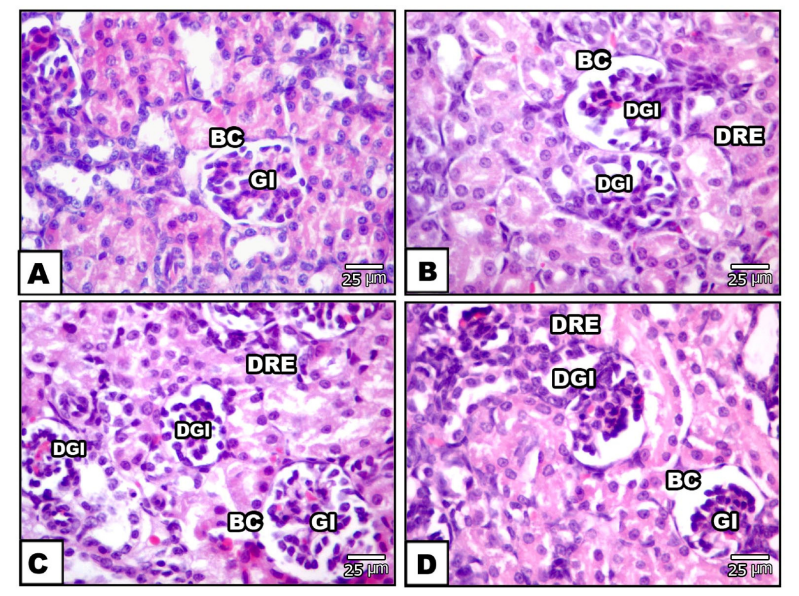

Fig. 2: Photomicrographs of histological sections of renal tissues of 14 days old rat pups. A: Control, B: Maternally Lipitor-treatment, C: Maternally hypercholesterolemic-treatment, D: Maternally hypercholesterolemic and Lipitor-treatment, Hx-E. Abbreviations: BC: Bowman's capsule, DGl: degenerated glomeruli, DRE: Degenerated renal epithelium; GI: Glomeruli

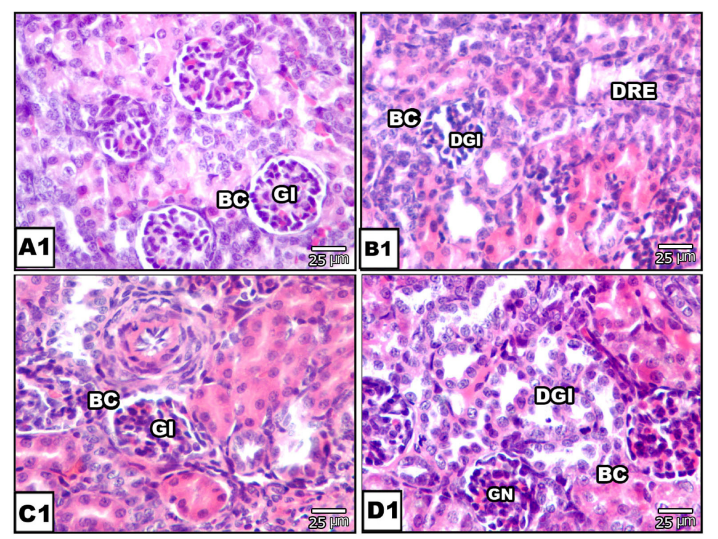

Fig. 3: Photomicrographs of histological sections of renal tissues of 21 days old rat pups. A: Control, B: Maternally Lipitor-treatment, C: Maternally hypercholestero-lemic-treatment, D: Maternally hypercholesterolemic and Lipitor-treatment. Hx-E. Abbreviations: BC: Bowman's capsule; DGl: degenerated glomeruli; DRE: Degenerated renal epithelium, Gl: Glomeruli

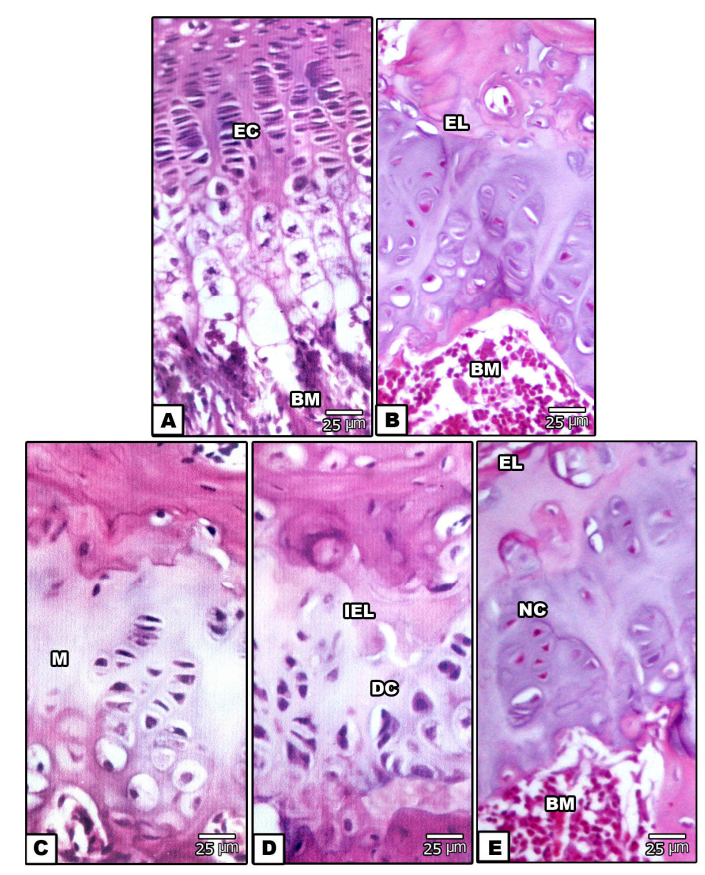

Fig. 4: Photomicrographs of longitudinal histological sections of epiphyseal cartilage of 21 days old offspring. A: Control showing normal ordinary arrangement of cartilage column, B: Lipitor treatment showing disorganized cartilage column and regenerated chondrocytes, C\&D: Hypercholesterolemic-treatment showing disorganized cartilage column and degenerated cartilage cells. E: Hypercholesterolemic and Lipitor treatment showing newly formed cartilage cells. H\&E. Abbreviations: BM: Bone marrow, DC: Degenerated chondrocytes, EC: epiphyseal cartilage, EL: epiphyseal line, IEL: irregular epiphyseal line, M: Matrix, NC: Normal cartilage 
cartilage column and widened of cartilage stromata. The observed increased damage and degeneration of cartilage cells and comparative reduction of epiphyseal cartilage may lead to softening of bone, decreased its growth rate and increased incidence of fractures. The present findings agreed with the work of Pirih et al. (2012) who reported increased generation of lipid oxidation products, in the subendothelial spaces of vasculature and bone associated with femoral bone strength and stiffness. Also, atherosclerosis was found to be associated with increased serum levels of parathyroid hormone, TNF-alpha, calcium and phosphorus and reduction of procollagen type I Nterminal propeptide, a serum marker of bone formation, in mice (Pirih et al., 2012).

Furthermore Lipitor-treated alone or in combination with hypercholesterolemia was found to improve femoral epiphysis and renal tissue to some extent and not equal to normal ones. The antilipidemic drug simvastatin (Soares et al., 2015) and atorvastatin (Rajamannan, 2015) were found to improve the impairing bone integrity related to bone fracture in mice as a result of hyperlipidemia.

Similar findings of deformities of epiphyseal cartilage was reported by El-Sayyad et al. (2010 \& 2015) who found reduction in long bone ossification and retarded differentiation of chondrification and osteoblast differentiation in rat foetus maternally fed on hypercholesterolemic diet.

\section{Conclusion}

Hypercholesterolemia altered renal morphology which might have affected synthesis of essential elements of bone formation leading to defective bone growth in their offspring. Lipitor-treatment improved maternal renal structure which also resulted in improved bone growth of their young.

\section{References}

Al-Rejaie SS, Abuohashish HM, Alkhamees OA, Aleisa AM, Abdulaziz S, Alroujayee AS (2012) Gender difference following high cholesterol diet induced renal injury and the protective role of rutin and ascorbic acid combination in Wistar albino rats. Lipids Health Dis 11: 41.

Andrade L, Campos SB, Seguro AC (1998) Hypercholesterolemia aggravates radiocontrast nephrotoxicity: protective role of L-arginine. Kidney Int 53: 1736-42.

Arnadottir M (2012) The question of primary lipid nephrotoxicity. Nephrol Dial Transplant 27: 26142615.

Badimon L, Vilahur G (2014) Thrombosis formation on atherosclerotic lesions and plaque rupture. J Intern Med 276: 618-32.
El-Sayyad HIH, El-Ghawet HA, Al-Haggar MS, Bakr EH (2015) Impairment of bone growth of wistar rat fetuses of diabetic and hypercholesterolemic mothers. Egypt J Basic Appl Sci 2: 1-12.

El-Sayyad HIH, El-Sherbiny MA, Sobh MA, Ibrahim M (2010) Protective effects of Morus alba leaves extract against congenital and skeletal malformations of diabetic and hypercholesterolemic mother albino rat's offspring. Egypt J Exp Biol (Zool) 6: 365-374.

Enkhmaa B, Shiwaku K, Anuurad E, Nogi A, Kitajima K, Yamasaki M, Oyunsuren T, Yamane Y (2005) Prevalence of the metabolic syndrome using the Third Report of the National Cholesterol Educational Program Expert Panel on Detection, Evaluation, and Treatment of High Blood Cholesterol in Adults (ATP III) and the modified ATP III definitions for Japanese and Mongolians. Clin Chim Acta 352: 105-13.

French SW, Yamanaka W, Ostwald R (1967) Dietary induced glomerulosclerosis in the guinea pig. Arch Pathol 83: 204-10.

Ghosh SS, Righi S, Krieg R, Kang L, Carl D, Wang J, Massey HD, Sica DA, Gehr TW, Ghosh S (2015) High fat high cholesterol diet (Western Diet) aggravates atherosclerosis, hyperglycemia and renal failure in nephrectomized LDL receptor Knockout Mice: Role of intestine derived lipopolysaccharide. PLoS One 10: e0141109.

Greco BA, Breyer JA (1997) Cholesterol as a predictor of progression in nondiabetic chronic renal disease. Contrib Nephrol 120: 48-61.

Gyebi L, Soltani Z, Reisin E (2012) Lipid nephrotoxicity: new concept for an old disease. Curr Hypertens Rep 14: 177-81.

Keane WF, Tomassini JE, Neff DR (2011) Lipid abnormalities in patients with chronic kidney disease. Contrib Nephrol 171: 135-142.

Kim HJ, Moradi H, Yuan J, Norris K, Vaziri ND (2009) Renal mass reduction results in accumulation of lipids and dysregulation of lipid regulatory proteins in the remnant kidney. Am J Physiol Renal Physiol 296: F1297-306.

Kivipelto M, Helkala EL, Laakso MP, Hanninen T, Hallikainen M, Alhainen K, Soininen H, Tuomilehto J, Nissinen A (2001) Midlife vascular risk factors and Alzheimer's disease in later life: longitudinal, population based study. BMJ 322: 1447-1451.

Maschio G, Oldrizzi L, Rugiu C, De Biase V, Loschiavo C (1991) Effect of dietary manipulation on the lipid abnormalities in patients with chronic renal failure. Kidney Int Suppl 31: S70-2.

Mori Y, Hirano T (2012) Ezetimibe alone or in combination with pitavastatin prevents kidney dysfunction in 5/6 nephrectomized rats fed highcholesterol. Metabolism 61: 379-88. 
Oberle GP, Niemeyer J, Thaiss F, Schoeppe W, Stahl RA (1992) Increased oxygen radical and eicosanoid formation in immune-mediated mesangial cell injury. Kidney Int 42: 69-74.

Oda H, Keane WF (1999) Recent advances in statins and the kidney. Kidney Int Supplm 71: S2-S5.

Pirih F, Lu J, Ye F, Bezouglaia O, Atti E, Ascenzi M G, Tetradis S, Demer LL, Aghaloo T, Tintut Y (2012) Adverse Effects of hyperlipidemia on bone regeneration and strength. J Bone Miner Res 27: 309-318.

Rajamannan NM (2015) Atorvastatin attenuates bone loss and aortic valve atheroma in LDLR mice. Cardiology 132: 11-5.

Sanz-Rosa D, Ruilope LM, Diaz C, Hernandez G, Lahera V, Cachofeiro V (2001) Atorvastatin prevents glomerulosclerosis and renal endothelial dysfunction in hypercholesterolaemic rabbits. Nephrol Dial Transplant 16(Suppl 1): 40-44.

Soares EA, Novaes RD, Nakagaki WR, Fernandes GJ, Garcia JA, Camilli JA (2015) Metabolic and structural bone disturbances induced by hyperlipidic diet in mice treated with simvastatin. Int J Exp Pathol 96: 261-8.

Sucajtys-Szulc E, Szolkiewicz M, Swierczynski J, Rutkowski B (2016) Up-regulation of liver Pcsk9 gene expression as a possible cause of hypercholesterolemia in experimental chronic renal failure. Mol Cell Biochem 411: 281-7.
Studer M, Briel M, Leimenstoll B, Glass TR, Bucher HC(2005) Effect of Different Antilipidemic Agents and Diets on Mortality. A Systematic Review. Arch Intern Med 165: 725-730.

Stulak JM, Lerman A, Caccitolo JA, Wilson SH, Romero JC, Schaff HV, Rodriguez Porcel M, Lerman LO (2001) Impaired renal vascular endothelial function in vitro in experimental hypercholesterolemia. Atherosclerosis 154: 195-201.

Tall AR, Yvan-Charvet L (2015) Cholesterol, inflammation and innate immunity. Nat Rev Immunol 15: 104-16.

Wolfort RM, Stokes KY, Granger DN (2008) CD4+ T lymphocytes mediate hypercholesterolemiainduced endothelial dysfunction via a NAD (P)H oxidase-dependent mechanism. Am J Physiol Heart Circ Physiol 294: H2619-26.

Yang DW, Jia RH, Yang DP, Ding GH, Huang CX (2004) Dietary hypercholesterolemia aggravates contrast media-induced nephropathy. Chin Med J (Engl). 117: 542-6.

Yang DW, Lin S, Yang DP, Wei L, Shang WY (2012) Effects of hypercholesterolemia on contrast mediainduced nephrotoxicity in rats. Zhonghua Yi Xue Za Zhi 92: 1424-7.

Zou JG, Wang ZR, Huang YZ, Cao KJ, Wu JM (2003) Effect of red wine and wine polyphenol resveratrol on endothelial function in hypercholesterolemic rabbits. Int J Mol Med 11: 317-320. 